\title{
Die nichtinvasive Beatmung bei Patienten mit stabiler, schwergradiger chronisch-obstruktiver Bronchitis und Lungenemphysem (COPD)
}

\author{
B. Schucher ${ }^{1}$ \\ J. Zerbst ${ }^{1}$ \\ H. J. Baumann ${ }^{2}$
}

Noninvasive Mechanical Ventilation in Patients with Stable Severe COPD

\section{Zusammenfassung}

Die nichtinvasive Beatmung hat sich in den letzten 15 Jahren zu einer unverzichtbaren Therapieform bei Patienten mit restriktiven thorakalen Erkrankungen wie der Kyphoskoliose, dem posttuberkulösem Syndrom und neuromuskulären Erkrankungen entwickelt. Bei Patienten mit einer schwergradigen chronisch obstruktiven Bronchitis und einem Lungenemphysem (COPD) ist der Nutzen für die akute ventilatorische Dekompensation im Rahmen einer Exazerbation sehr gut belegt und gilt mittlerweile als Standardtherapie. Die nichtinvasive Beatmung wird jedoch auch in zunehmendem Maße bei Patienten mit einer chronisch ventilatorischen Insuffizienz eingesetzt, ist hier aber umstritten. Die nichtinvasive Beatmung führt hier zu einer deutlichen Reduktion der Atemarbeit der stark belasteten Atemmuskulatur, dies führte jedoch meist zu keiner Verbesserung der maximalen Inspirationskraft $\left(\mathrm{Pi}_{\max }\right)$. Eine Verbesserung der Schlafqualität konnte insbesondere in Studien nachgewiesen werden, in denen höhere effektive Beatmungsdrücke verwendet wurden. Eine Verbesserung der Gehstrecke ist bisher nur in Kurzzeitstudien belegt. Ein Anstieg der Lebensqualität ist hingegen in fast allen Studien nachweisbar. Eine Senkung der Mortalität konnte dagegen bisher nicht sicher bewiesen werden. Nach der vorliegenden Studienlage sollten bevorzugt Patienten mit einer stark ausgeprägten Hyperkapnie ausgewählt werden, es sollten überwiegend Beatmungsverfahren Anwendung finden, die hohe effektive Beat-

\section{Abstract}

Noninvasive positive pressure ventilation in patients with stable chronic obstructive pulmonary disease. The role of non-invasive positive pressure ventilation (NIPPV) is well documented in patients with restrictive thoracic diseases like kyphoscoliosis, tuberculosis sequelae or neuromuscular disease. There is also a good evidence for the use of NIPPV in acute respiratory failure in patients with an exacerbation of COPD. The application of NIPPV in patients with chronic respiratory failure is growing, but there is less evidence than in restrictive disorders. NIPPV can unload the respiratory muscles in patients with chronic hypercapnic COPD and so alleviates fatigue of the respiratory pump, but improvement in the maximal inspiratory pressure $\left(\mathrm{Pi}_{\max }\right)$ is small or even absent. An improvement of sleep quality has also postulated, there was an increase in total sleep time and sleep effectiveness when using higher inspiratory pressure. An increase of the walking distance was shown in short term studies, only. In most studies, there was an increase in quality of life as a main topic. Mortality was unchanged in the two longterm randomised controlled studies. Current data suggest a possible role of NIPPV in patients with severe hypercapnia. A high effective inspiratory pressure and a ventilator mode with a significant reduction in the work of breathing should be choosen. NIPPV should be started in hospital, a close reassessment must

Institutsangaben

${ }^{1}$ Krankenhaus Großhansdorf, Zentrum für Pneumologie und Thoraxchirurgie (Ärztlicher Direktor

Prof. Dr. med. H. Magnussen), Großhansdorf

${ }^{2}$ Universitätsklinikum Hamburg Eppendorf, Medizinische Klinik und Poliklinik I - Bereich Pneumologie

(Direktor Prof. Dr. med. H. Greten), Hamburg

Widmung

Herrn Prof. Dr. med. H. Magnussen zum 60. Geburtstag gewidmet

Korrespondenzadresse

Dr. med. Bernd Schucher · Krankenhaus Großhansdorf · Zentrum für Pneumologie und Thoraxchirurgie Wöhrendamm 80·22927 Großhansdorf ·E-mail: bhschucher@aol.com

Eingang: 10. April $2004 \cdot$ Nach Revision angenommen: 13. Mai 2004

Bibliografie

Pneumologie 2004; 58: 428-434 • (c) Georg Thieme Verlag KG Stuttgart • New York

DOI $10.1055 / \mathrm{s}-2004-818499$

ISSN 0934-8387 
mungsdrücke ermöglichen und somit eine vollständige Entlastung der Atemmuskulatur erreichen. Unter diesen Vorraussetzungen ist es möglich, einen großen Teil der sorgfältig ausgewählten und unter klinischen Bedingungen an die Beatmung adaptierten Patienten erfolgreich in eine Langzeittherapie zu überführen. Patienten, die die Beatmung in der Klinik akzeptieren zeigen im weiteren Verlauf eine hohe Therapiecompliance gemessen an der dokumentierten täglichen Nutzungsdauer. be performed. Patients who accepted NIPPV in the first weeks had a good compliance for long-term use.
Die chronisch obstruktive Bronchitis und das Lungenemphysem (COPD) sind Erkrankungen, die zu den führenden Todesursachen gehören. Ihre Prävalenz wird in der Zukunft noch zunehmen. Trotz einer optimalen medikamentösen Therapie leiden viele Patienten unter Belastungs- oder gar Ruhedyspnoe mit einer entsprechenden Einschränkung der körperlichen Belastbarkeit und der Lebensqualität.

Der Nutzen einer pneumologische Rehabilitation bei Patienten mit einer COPD ist gut belegt, es kann eine Verbesserung der Dyspnoe, der körperlichen Belastbarkeit und der Lebensqualität erreicht werden [1 -4]. Bei einem Teil der Patienten mit einer klinisch relevanten Hyperkapnie und somit einem Schweregrad IV der GOLD-Leitlinien ist jedoch allein durch eine pneumologische Rehabilitation keine Verbesserung erreichbar [5].

Aufgrund der ausgesprochen guten klinischen Ergebnisse der nichtinvasiven Beatmung bei hyperkapnischen Patienten mit einer restriktiven Ventilationsstörung wie der Kyphoskoliose, dem posttuberkulösem Syndrom oder neuromuskulären Erkrankungen $[6,7]$ wurde bereits frühzeitig versucht, die nichtinvasive Beatmung auch bei Patienten mit einer COPD und Hyperkapnie einzusetzen.

Bei der akuten ventilatorischen Insuffizienz aufgrund einer Exazerbation der COPD konnte durch eine Reihe von randomisierten Studien im Vergleich zur Standardtherapie ein eindeutiger Überlebensvorteil durch die Anwendung der nichtinvasiven Beatmung nachgewiesen werden und gilt daher mittlerweile als neue Standardtherapie [8 - 10].

Die nichtinvasive Beatmung wird auch bei Patienten mit einer stabilen Hyperkapnie eingesetzt, ist aber in dieser Indikationsstellung umstritten. Während in den GOLD-Leitlinien aus dem Jahr 2003 keine Indikation bei stabiler Hyperkapnie gesehen wird, empfehlen die deutschen Leitlinien [11] bei Patienten mit einer ausgeprägten Hyperkapnie am Tage den Einsatz der nichtinvasiven Beatmung (Heimbeatmung). Eine generelle Empfehlung wird jedoch noch nicht ausgesprochen.

Ursächlich für das ablehnende Statement der GOLD-Leitlinien waren eine Reihe von randomisierten Studien, die im Vergleich zu den nur konservativ behandelten Patienten keinen nachweisbaren Vorteil für die beatmete Gruppe erbringen konnten. Es gibt jedoch auch einige randomisierte und eine Reihe von nicht randomisierten Studien, die positive Ergebnisse zeigen konnten. Im Folgenden soll daher die Grundlage der nichtinvasiven Beat- mung bei COPD dargestellt werden, anschließend werden die vorliegenden Studienergebnisse kritisch interpretiert.

\section{Physiologische Grundlagen der nichtinvasiven Beatmung}

Hyperkapnische Patienten mit einer schweren COPD weisen in der Regel eine erhebliche Belastung ihrer Inspirationsmuskulatur auf. Ursachen sind neben der Obstruktion u.a. die erhöhte Totraumventilation und aufgrund der Überblähung ein ungünstiger Wirkungsbereich des Zwerchfells und der Interkostalmuskulatur [12]. Hieraus resultiert eine chronische Überlastung und Erschöpfung der Atempumpe, die als eine der Hauptursachen der Hyperkapnie angesehen wird [13].

Während des Schlafes kommt es zu einer weiteren Zunahme der Hypoventilation, insbesondere im REM-Schlaf. Die Ursache ist zumeist eine Abnahme des Atemzugvolumens und nicht das Auftreten von Apnoen [14]. Zusätzlich ist der Atemantrieb auf $\mathrm{CO}_{2}$ in der Nacht vermindert [15], der Widerstand der oberen Atemwege nimmt zu. Dies führt in der Summe zu einer weiteren Verschlechterung der Hyperkapnie und der Hypoxie im Schlaf. Die nächtliche Hypoxie kann zwar durch eine Sauerstoffgabe ausgeglichen werden, dies führt aber oft zu einer Zunahme der Hyperkapnie, insbesondere in den frühen Morgenstunden [16]. Die Hyperkapnie kann wiederum eine weitere Verschlechterung der Zwerchfellfunktion nach sich ziehen [17].

Es erscheint daher pathophysiologisch sinnvoll, bei Patienten mit COPD eine Entlastung der Atemmuskulatur und eine Verbesserung der nächtlichen Ventilation anzustreben.

\section{Entlastung der Atemmuskulatur unter nichtinvasiver Beatmung}

Eine Reihe von Studien haben die Effekte der nichtinvasiven Beatmung auf die Atemmuskulatur untersucht. Zunächst wurde die Auswirkung der Negativdruckbeatmung auf die Reduktion der Atemmuskelaktivität untersucht, es zeigte sich eine deutliche Abnahme der Zwerchfell-EMG-Aktivität während der Beatmung [18-20]. Die Negativdruckbeatmung spielt jedoch als Dauertherapie keine Rolle mehr, u. a. aufgrund der unkomfortablen Anwendung.

Die Reduktion der Atemmuskelaktivität ist auch für die Positivdruckbeatmung beschrieben [19,21 - 26]. Unter einer kontrollierten Beatmung mit Volumenvorgabe gelang es, die Aktivität des 
Zwerchfell-EMGs um 90\% zu reduzieren [25]. Wird stattdessen ein assistierter Beatmungsmodus gewählt, so ist die Reduktion der Zwerchfell-EMG-Aktivität nicht so stark ausgeprägt [24]. Werden bei assistierter Beatmung nur niedrige Beatmungsdrücke verwendet, so ist die Reduktion nicht mehr nachweisbar [22].

Diese Ergebnisse werden auch durch andere Untersuchungstechniken bestätigt. Bei ventilatorisch insuffizienten Patienten gelingt es unter einer kontrollierten Beatmungsform, den Gesamtsauerstoffverbrauch im Vergleich zur Spontanatmung um $25 \pm$ $7 \%$ zu reduzieren. Dies entspricht dem Sauerstoffverbrauch der Atemmuskulatur. Wird hingegen ein assistierter Modus gewählt, so ist lediglich eine Reduktion um $11 \pm 8 \%$ nachweisbar [27].

Auch die Atemarbeit kann bei hyperkapnischen Patienten mit COPD durch die Anwendung von Beatmungsformen mit hohen Beatmungsdrücken signifikant gesenkt werden, das Ausmaß der Absenkung korreliert mit dem effektiven Beatmungsdruck. Bei gleichem inspiratorischem Spitzendruck führte die Zugabe eines positiv endexspiratorischen Druckes (PEEP) zu einer Abnahme des Effektes, unter der Anwendung des CPAP-Modus war in einer Arbeit von Vanpee u. Mitarb. [28] keine Abnahme der Atemarbeit mehr zu beobachten. Entscheidend ist der effektive Beatmungsdruck (Differenz zwischen dem Druck inspiratorisch und exspiratorisch) [28].

Die hier beschriebenen Effekte auf die Entlastung der Atemmuskulatur führten jedoch lediglich in einer Studie zu einer dauerhaften Zunahme der maximalen Inspirationskraft [29], in den anderen Studien war keine Veränderung oder nur ein Trend zur Verbesserung nachweisbar [24,30-36]. Es muss jedoch kritisch hinterfragt werden, ob die maximale Inspirationskraft der richtige Parameter zur Beurteilung der Verbesserung der Atempumpfunktion ist. Möglicherweise wären Untersuchungen zur Ausdauerfähigkeit der Atempumpe ein besseres Maß, entsprechende Ergebnisse liegen aber nicht vor.

\section{Veränderungen der Schlafes}

Veränderungen der Schlafes unter der nichtinvasiven Beatmung wurden in verschiedenen kontrollierten Studien untersucht [30,31,33,37-39]. Die Ergebnisse sind jedoch nicht einheitlich und können nicht unabhängig von der Patientenselektion und der Beatmungsart betrachtet werden.

In der ersten kontrollierten randomisierten Studie zur nichtinvasiven Beatmung bei stabiler COPD von Strumpf u. Mitarb. [30] kam es in der Beatmungsgruppe in der Tendenz zu einer Abnahme der totalen Schlafzeit und der Schlafeffizienz, allerdings waren nur 7 Patienten in der Beatmungsgruppe und 6 in der Kontrollgruppe. Lin [31] konnte in einem Crossover-Design eine Zunahme des Arousal- und des Movementindex unter der Beatmung beobachten. Beide Studien zeichneten sich jedoch durch eine ausgesprochen schlechte Toleranz der Beatmung mit einer hohen Abbrecherquote aus. Bei Strumpf u. Mitarb. war der überwiegende Anteil der beatmeten Patienten nicht hyperkapnisch und würde somit nach den heute akzeptierten Kriterien keine Beatmungsindikation haben [40]. Lin beatmete mit niedrigen effektiven Beatmungsdrücken und ohne PEEP trotz Verwendung eines BiPAP-Beatmungssystems, so dass eine unzureichende $\mathrm{CO}_{2}$-Elimination vermutet werden darf [41].

In der randomisierten Studie von Garrod u. Mitarb. [29] wurden überwiegend normokapnische Patienten ohne schwergradige nächtliche Hypoventilationen beatmet, so dass wie erwartet eine Verbesserung der Schlafqualität nicht auftrat.

Hingegen konnten Elliott u. Mitarb. [37] eine Verbesserung der nächtlichen Oxygenierung, eine Absenkung des $\mathrm{PCO}_{2}$, eine $\mathrm{Zu}$ nahme der totalen Schlafzeit und der Schlafeffizienz zeigen. In dieser Studie wurde eine assistiert-kontrollierte Beatmung mit Volumenvorgabe angewendet, das Atemminutenvolumen lag im Median bei 23 Litern, der Beatmungsdruck erreichte Werte von $30-40 \mathrm{cmH}_{2} \mathrm{O}$.

Auch in der kontrollierten randomisierten Studie von Meecham Jones u. Mitarb. [38] konnte sowohl eine Zunahme der totalen Schlafzeit als auch der Schlafeffizienz im Vergleich zur alleinigen Sauerstofftherapie nachgewiesen werden. Die Abbrecherrate in dieser Studie war gering, die Patienten waren bei Einschluss in die Studie deutlich hyperkapnisch, der effektive Beatmungsdruck war mit $18 \mathrm{cmH}_{2} \mathrm{O}$ höher. In der bislang größten randomisierten Studie zeigte sich ein Trend zu einer besseren Schlafqualität, wenn auch nur nach den subjektiven Angaben der Patienten auf einer semiqualitativen Skala [33].

\section{Körperliche Belastbarkeit}

Nur wenige Studien haben Veränderungen der Gehstrecke untersucht. Renston u. Mitarb. [24] konnte nach 5 Tagen Beatmung am Tage eine signifikante Zunahme im 6-Minuten-Gehtest von 780 auf 888 Fuß in der Beatmungsgruppe nachweisen, in der Kontrollgruppe trat keine Veränderung auf. Garrod u. Mitarb. [29] untersuchten die Wirkungen der Beatmung zusätzlich zu einem Trainingprogramm und konnten nach 8 Wochen eine Verbesserung im Vergleich zur Kontrollgruppe im Shuttle-Walking-Test dokumentieren, allerdings wurden normokapnische Patienten beatmet, die tägliche Nutzung der Beatmung war mit nur $2 \mathrm{~h} / \mathrm{d}$ im Median gering. In 2 Studien mit längeren Beobachtungsdauern von 3 bzw. 24 Monaten $[33,38]$ konnte keine Verbesserung erzielt werden.

\section{Lebensqualität}

Die Verbesserung der Lebensqualität ist ein Hauptanliegen bei der Behandlung von Patienten mit schwergradiger COPD und gilt als Erfolgsparameter, der noch vor der Reduktion der Mortalität rangiert [42,43]. Die Messung der gesundheitsbezogenen Lebensqualität ist jedoch methodisch nicht ohne Schwierigkeiten. In der Regel erfolgt dies über krankheitsübergreifende oder krankheitsspezifische Fragebogen [44]. Verwendet wurden der krankheitsübergreifende Fragebogen MOS 36-Item Short-Form Heath Survey (SF36) [45-47] und die krankheitsspezifischen Fragebogen St. George's Respiratory Questionnaire (SGRQ) $[33,38,48]$ und der Chronic Respiratory Disease Questionnaire (CRQ) [29]. Bei Clini u. Mitarb. [33] wurde zusätzlich ein speziell für Patienten mit einer chronischen respiratorischen Insuffizienz 
entwickelter Fragebogen eingesetzt, der Maugeri Foundation Respiratory item set (MRF-28). In allen Studien konnte eine Verbesserung der Lebensqualität nachgewiesen werden, dies gilt auch für die randomisierten Studien $[29,33,38]$. Seit kurzem steht für Patienten mit einer ventilatorischen Insuffizienz ein deutscher krankheitsspezifischer Fragebogen zur Verfügung, der Severe Respiratory Insufficiency (SRI) Questionnaire (49). Er eignet sich insbesondere, wenn Patienten mit verschiedenen Grundkrankheiten vergleichend betrachtet werden.

\section{Mortalität}

Während es hinreichende Daten zur Verbesserung der Lebensqualität gibt, ist die Datenlage hinsichtlich der Mortalität deutlich geringer. Nur 2 Studien untersuchten prospektiv randomisiert mit Kontrollgruppe den Einfluss der Heimbeatmung auf das Überleben. Weder in der Studie von Casanova u. Mitarb. [32], die den Zeitraum von einem Jahr untersuchte, noch in der Studie von Clini u. Mitarb. [33] (Zeitraum zwei Jahre) konnte ein Verringerung der Mortalität dokumentiert werden. Kritisch angemerkt werden muss jedoch, dass in beiden Studien nur teilentlastende Beatmungsverfahren mit niedrigen effektiven Beatmungsdrücken verwendet wurden, und dass Casanova u. Mitarb. auch normokapnische Patienten eingeschlossen haben.

Aber auch die in den Studien ohne Kontrollgruppe hilfsweise durchgeführten Vergleiche mit historischen Kontrollkollektiven, hier insbesondere die beiden Studien zur Langzeitsauerstofftherapie [50,51], erbrachten keinen klaren Vorteil.

Eine Reduzierung der Mortalität konnte somit bisher nicht nachgewiesen werden. Ob dies in der geplanten deutschen Multicenterstudie der Arbeitsgemeinschaft Heimbeatmung und Respiratorentwöhnung e.V. gelingen wird, bleibt abzuwarten. In dieser Untersuchung sollen im Gegensatz zu den bisherigen Studien hohe effektive Beatmungsdrücke verwendet werden, um eine effektive Reduktion des $\mathrm{PCO}_{2}$ zu erreichen.

\section{Pulmonalarteriendruck}

Schönhofer u. Mitarb. untersuchten die Langzeitwirkung der nichtinvasiven Beatmung auf die pulmonale Hämodynamik [52]. Nach einen Jahr Beatmungstherapie konnte zwar bei Patienten mit thorakal-restriktiven Erkrankungen der mittlere pulmonale Druck signifikant von $33,2 \pm 10$ auf $24,8 \pm 6,2 \mathrm{mmHg}$ gesenkt werden, bei den Patienten mit schwergradiger COPD blieb der pulmonale Druck jedoch mit 25,3 $\pm 6,0$ und $27,5 \pm 6,0 \mathrm{mmHg}$ unverändert, obwohl es in beiden Gruppen zu einer signifikanten Verbesserung des $\mathrm{PO}_{2}$ und des $\mathrm{PCO}_{2}$ gekommen war. Allerdings muss angemerkt werden, dass nur wenige Patienten mit COPD vor Beatmungsbeginn einen pulmonalen Hypertonus aufwiesen und somit, wie auch von den Autoren diskutiert, wenig Spielraum für eine Verbesserung vorhanden war.

\section{Patientenselektion und Beatmungsparameter}

Die uneinheitliche Studienlage spiegelt sich auch in einer nicht einheitlichen Patientenselektion und unterschiedlichen Beatmungsformen und -einstellungen wieder. Eine generelle Aussage zur Wirksamkeit oder Unwirksamkeit der Beatmung lässt sich daraus nicht ableiten. Zwar wurde der Versuch einer Metaanalyse unternommen [53], deren Nutzen bleibt aber bei insgesamt nur 4 eingeflossenen Studien $[30,32,38,39]$ mit summativ 58 Beatmungspatienten fragwürdig, zumal in 2 dieser Studien die mittlerweile allgemein als Eingangsvoraussetzung geforderte Hyperkapnie [40] kein Einschlusskriterium war, und in 3 Studien sehr niedrige Beatmungsdrücke verwendet wurden. Auch wurde die bisher größte Studie [33] aufgrund des sich überschneidendenden Erscheinungsdatums nicht berücksichtigt.

Wenn man die Eckpunkte Hyperkapnie und Beatmungsparameter der randomisierten und nicht randomisierten Studien in einer Tabelle zusammenführt (Tab.1), so zeigt sich, dass in den Studien ohne positive Effekte die Hyperkapnie weniger stark ausgeprägt war als bei den positiven Studien. Auch war der effektive Beatmungsdruck in den positiven Studien deutlich höher als in den negativen Studien. Wie bereits dargestellt, bestimmt aber die Höhe des effektiven Beatmungsdruckes die Verbesserung der Ventilation $[28,41]$ und somit auch den Rückgang der Hyperkapnie während der Beatmung. Dies ist wiederum notwendig, um eine Verbesserung der Hyperkapnie am Tage zu erreichen. Windisch u. Mitarb. konnten zeigen, dass der $\mathrm{PCO}_{2}$ unter Spontanatmung mit den $\mathrm{PCO}_{2}$ unter Beatmung korreliert. Je niedriger der $\mathrm{PCO}_{2}$ unter Beatmung, desto niedriger ist dann auch der $\mathrm{PCO}_{2}$ unter Spontanatmung [36]. In einer Analyse der eigenen Daten konnten wir belegen, dass die Absenkung des $\mathrm{PCO}_{2}$ unter Spontanatmung nach 3 Monaten mit der Absenkung des $\mathrm{PCO}_{2}$ während der Beatmung in Kombination mit der durchschnittlichen täglichen Beatmungszeit korreliert [35] (Abb.1).

Unklar bleibt aber weiterhin die langfristige Bedeutung des Rückganges der Hyperkapnie.

\section{Compliance}

Die nasale oder oronasale Beatmung mit einer Maske ist eine Behandlungsform, die dem Patienten unabhängig vom klinischen Nutzen auch mit Nebenwirkungen und Dyscomfort belastet. Ernste Nebenwirkungen sind zwar eine Rarität, aber leichte Nebenwirkungen wie Druckstellen durch die Maske oder ausgetrocknete oronasale Schleimhäute treten häufig auf $[6,54]$. Aufgrund dieser unangenehmen Begleitumstände und der erforderlichen langen täglichen Benutzungsdauer ist eine langfristige Compliance des Patienten nur dann zu erwarten, wenn der subjektive Nutzen der Beatmung die Unannehmlichkeiten zumindest aufwiegt. Im Gegensatz zur medikamentösen Therapie bietet die Beatmungstherapie den Vorteil einer guten Complianceüberprüfung mittels des geräteinternen Stundenzählers.

Die vorliegenden Studiendaten zeigen eine hohe durchschnittliche tägliche Nutzung der Beatmung, sie lag in der Regel im Mittel über 6 Stunden pro Tag $[30,32,33,36,38,45,48,55]$. In einer eigenen Studie stieg die Beatmungszeit von 7,0 $\pm 3,6 \mathrm{~h} / \mathrm{d}$ nach 3 
Tab. 1 Zusammenstellung der randomisierten und einiger nicht kontrollierter Studien zur Beatmung bei COPD mit wichtigen Eckpunkten. Die Patientenanzahl bezieht sich auf die erfolgreich beatmeten und in die in das Studienergebnis eingeschlossenen Patienten.

\begin{tabular}{|c|c|c|c|c|c|c|}
\hline & Patienten & $\begin{array}{l}\mathrm{PaCO}_{2} \\
\text { Beginn } \\
(\mathrm{mmHg})\end{array}$ & $\begin{array}{l}\mathrm{PaCO}_{2} \\
\text { im Verlauf } \\
(\mathrm{mmHg})\end{array}$ & $\begin{array}{l}\text { FEV }_{1} \\
\text { (I) }\end{array}$ & $\begin{array}{l}\text { Beatmungsdruck } \\
\text { absolut } \\
\left(\mathrm{cmH}_{2} \mathrm{O}\right)\end{array}$ & $\begin{array}{l}\text { effektiv } \\
\left(\mathrm{cm} \mathrm{H}_{2} \mathrm{O}\right)\end{array}$ \\
\hline & \multicolumn{6}{|c|}{ kontrollierte randomisierte Studien ohne positive Ergebnisse } \\
\hline Strumpf [30] & 7 & 46 & 50 & 0,54 & $15 / 2$ & 13 \\
\hline Lin [31] & 12 & 50 & 50 & $33 \%$ & $12 / 1$ & 11 \\
\hline Gay [39] & 7 & 55 & & 0,62 & $10 / 2$ & 8 \\
\hline \multirow[t]{2}{*}{ Casanova [32] } & 20 & 51 & 51 & 0,82 & $12 / 4$ & 8 \\
\hline & \multicolumn{6}{|c|}{ kontrollierte randomisierte Studien mit teilweise positiven Ergebnissen } \\
\hline \multirow[t]{2}{*}{ Clini [33] } & 39 & 53 & 51 & $27 \%$ & $14 / 2$ & 12 \\
\hline & \multicolumn{6}{|c|}{ kontrollierte randomisierte Studien mit überwiegend positiven Ergebnissen } \\
\hline Meecham Jones [38] & 14 & 56 & 53 & 0,86 & $18 / 2$ & 16 \\
\hline \multirow[t]{2}{*}{$\operatorname{Diaz}[26]$} & 18 & 56 & 48 & 0,72 & $18 / 2$ & 16 \\
\hline & \multicolumn{6}{|c|}{ nicht kontrollierte Studien mit überwiegend positiven Ergebnissen } \\
\hline Elliott [34] & 8 & 60 & 54 & 0,54 & Volumenvorgabe & $30-40$ \\
\hline Sivasothy [45] & 26 & 63 & 56 & 0,68 & n.a. & n.a. \\
\hline Schucher [35] & 41 & 57 & 49 & 0,72 & 21 & 21 \\
\hline Windisch [36] & 14 & 60 & 46 & 0,97 & 30 & 30 \\
\hline
\end{tabular}

Monaten auf 7,7 \pm 4,2 h/d nach einem Jahr und 9,5 $\pm 4,4 \mathrm{~h} / \mathrm{d}$ nach 2 Jahren Beatmungsdauer [35].

Somit ist bei Patienten, die primär an die Beatmung adaptiert werden können eine gute Therapiecompliance belegt. Die Verbesserungen der Beatmungsgeräte und der verfügbaren Masken sowie die Auswahl der geeigneten Patienten haben hierzu beigetragen. Eine sichere Vorabeinteilung in Responder und Non-Responder ist jedoch nach derzeitigem Kenntnisstand nicht möglich. Schönhofer u. Mitarb. konnten in einer frühen Studie eine Responderrate von $70 \%$ belegen, wobei ledig $14 \%$ der Patienten eine generelle Inakzeptanz zeigten, weitere $16 \%$ akzeptierten die Beatmung zwar, es ließ sich aber keine objektive oder subjektive Verbesserung erreichen [56].

\section{Zusammenfassung und Ausblick}

Zum jetzigen Zeitpunkt ist die Bedeutung der nichtinvasiven Beatmung als Dauertherapie bei Patienten mit stabiler schwergradiger COPD noch nicht ausreichend wissenschaftlich belegt. Nach den vorliegenden Studiendaten kann weder eine generelle Empfehlung ausgesprochen werden, noch liegt ein Unwirksamkeitsnachweis vor. Die genaue Analyse der Studienlage gibt jedoch Hilfestellung bei der Auswahl der potenziellen Beatmungspatienten. Das Ausmaß der Hyperkapnie scheint hier der wesentliche Faktor. Dies wurde im Consensus Conference Report [40] als Indikation zur Beatmung zusammengefasst:

1. Symptome der ventilatorischen Insuffizienz wie Müdigkeit, Dyspnoe, morgendliche Kopfschmerzen etc.


(PaCO2 spont. - PaCO2 NIPPV) * tägl. Nutzung NIPP
Abb. 1 Die Differenz zwischen dem initialen $\mathrm{PaCO}_{2}$ unter Spontanatmung und dem $\mathrm{PaCO}_{2}$ unter der Beatmung als Produkt mit der täglichen Nutzungsdauer korreliert signifikant mit dem Absinken des $\mathrm{PaCO}_{2}$ unter Spontanatmung nach 3 Monaten [35]. 
2. a) Hyperkapnie ab $55 \mathrm{mmHg}$

b) Hyperkapnie zwischen 50 und $54 \mathrm{mmHg}$ und nächtliche Entsättigungen über mindesten 5 Minuten $<88 \%$ trotz der Gabe von 2 1/min Sauerstoff

c) Hyperkapnie zwischen 50 und $54 \mathrm{mmHg}$ und mindesten 2 Hospitalisationen pro Jahr aufgrund von hyperkapnischen Exazerbationen.

Nach eigenen Erfahrungen kann in Einzelfällen mit einer ausgeprägten Symptomatik auch von diesen Grenzen abgewichen werden, allerdings war bei vollständig normokapnischen Patienten in keinem Fall eine klinisch relevante und dauerhafte Besserung zu erzielen.

Im Consensus Report [40] wird in Hinblick auf Patienten mit COPD keine Empfehlung zur Beatmungseinstellung gegeben, es wird jedoch die Abhängigkeit der Effektivität vom effektiven Beatmungsdruck diskutiert. Nach derzeitiger Datenlage scheinen höhere effektive Beatmungsdrücke zu besseren klinischen Ergebnissen zu führen. Auch gibt es Hinweise dafür, dass vollständig entlastende Beatmungsverfahren Vorteile gegenüber teilentlastenden Verfahren haben.

Bis zur weiteren wissenschaftlichen Klärung sollte die Heimbeatmung bei dieser Patientengruppe daher nur nach sorgfältiger Prüfung und in Anlehnung an die obengenannten Kriterien durchgeführt werden. Eine kritische Beobachtung des individuellen Therapieerfolges und auch der Therapiecompliance ist unabdingbar. Zur Vermeidung von unnötigen hohen Kosten ist es ratsam, nach einer intensiven klinischen Adaptation an die Beatmung zunächst eine Probetherapie für 1-2 Monate anzuschließen, erst danach sollte über eine Langzeittherapie entschieden werden [43].

\section{Literatur}

${ }^{1}$ Lacasse Y, Wong E, Guyatt GH et al. Meta-analysis of respiratory rehabilitation in chronic obstructive pulmonary disease. Lancet 1996; 348: $1115-1119$

${ }^{2}$ Goldstein RS, Gort EH, Stubbing D et al. Randomised controlled trial of respiratory rehabilitation. Lancet 1994; 344: 1394-1397

${ }^{3}$ Griffiths TL, Burr ML, Campell IA et al. Results at 1 year of outpatient multidisciplinary pulmonary rehabilitation: a randomised controlled trial. Lancet 2000; 355: $362-368$

${ }^{4}$ Wijkstra PJ, Altena R van, Kran J et al. Quality of life in patients with chronic obstructive pulmonary disease improves after rehabilitation at home. Eur Respir J 1994; 7: 269-273

${ }^{5}$ Wedzicha JA, Bestall JC, Garrod R et al. Randomized controlled trial of pulmonary rehabilitation in severe chronic obstructive pulmonary disease patients, stratified with the MRC scale. Eur Respir J 1998; 12: $363-369$

${ }^{6}$ Leger P, Bedicam JM, Cornette A et al. Nasal intermittent positive pressure ventilation. Long-term follow-up in patients with severe chronic respiratory insufficiency. Chest 1994; 105: 100-105

${ }^{7}$ Simonds AK, Elliott MW. Outcome of domiciliary nasal intermittent positive pressure ventilation in restrictive and obstructive disorders. Thorax 1995; 50: 604-609

${ }^{8}$ Bott J, Carroll MP, Conway JH et al. Randomised controlled trial of nasal ventilation in acute ventilatory failure due to chronic obstructive airways disease. Lancet 1993; 341: $1555-1557$

${ }^{9}$ Brochard L, Mancebo J, Wysocki M et al. Noninvasive ventilation for acute exacerbation of chronic obstruktive pulmonary disease. N Eng J Med 1995; 333: 817-822
${ }^{10}$ Kramer N, Meyer TJ, Meharg J et al. Randomized, prospective trial of noninvasive positive pressure ventilation in acute respiratory failure. Am J Respir Crit Care Med 1995; 151: 1799-1806

${ }^{11}$ Worth H, Buhl R, Cegla U et al. Leitlinie der Deutschen Atemwegsliga und der Deutschen Gesellschaft für Pneumologie zur Diagnostik und Therapie von Patienten mit chronisch obstruktiver Bronchitis und Lungenemphysem (COPD). Pneumologie 2002; 56: 704-738

${ }^{12}$ Braun NMT, Rochester DF. Respiratory muscle function in chronic obstructive pulmonary disease (COPD). Am Rev Respir Dis 1997; 115: 91

${ }^{13}$ Roussos C, Macklem PT. The respiratory muscles. N Eng J Med 1982; 307: $786-797$

14 Wedzicha JA, Muir JF. Noninvasive ventilation in chronic obstructive pulmonary disease, bronchiectasis and cystic fibrosis. Eur Respir J 2002; 20: 777-784

${ }^{15}$ Ingrassia TS, Nelson SB, Harris CD et al. Influence of sleep state on $\mathrm{CO}_{2}$ responsiveness. Am Rev Respir Dis 1994; 144: 1125 - 1129

${ }^{16}$ Goldstein RS, Ramcharan V, Bowes G et al. Effect of supplemental nocturnal oxygen on gas exchange in patients with severe obstructive lung disease. N Eng J Med 1994; 310: 425-429

${ }^{17}$ Juan G, Calverley P, Talamo $C$ et al. Effect of carbon dioxide on diaphragmatic function in human beings. N Eng J Med 1984; 310: $874-879$

${ }^{18}$ Rochester DF, Braun NMT, Laine S. Diaphragmatic energy expenditure in chronic respiratory failure. The effect of assisted ventilation with body respirators. Am J Med 1977; 63: 223 - 232

${ }^{19}$ Belman MJ, Hoo GWS, Kuei JH et al. Efficacy of positive vs negative pressure ventilation in unloading the respiratory muscle. Chest 1990; 98: $850-856$

${ }^{20}$ Nava S, Ambrosino N, Zocchi L et al. Diaphragmatic rest during negative pressure ventilation by Pneumowrap. Assessment in normal and COPD patients. Chest 1990; 98: 857-865

${ }^{21}$ Carrey Z, Gottfried SB, Levy RD. Ventilatory muscle support in respiratory failure with nasal positive pressure ventilation. Chest 1990; 97: $150-158$

${ }^{22}$ Lien TC, Wang JH, Chang MT et al. Comparsion of BiPAP nasal ventilation and Ventilation via iron lung in servere stable COPD. Chest 1993; 104: $460-466$

${ }^{23}$ Nava S, Ambrosino N, Rubini F et al. Effect of nasal pressure support ventilation and external PEEP on diaphragmatic activity in patients with severe stable COPD. Chest 1993; 103: 143-150

${ }^{24}$ Renston JP, DiMarco AF, Supinski GS. Respiratory muscle rest during nasal BiPAP ventilation in patients with stable severe COPD. Chest 1994; 105: 1053 - 1060

${ }^{25}$ Jimenez JFM, de Cos Escuin JS, Vicente CD et al. Nasal intermittent positiv pressure ventilation - Analysis of its withdrawal. Chest 1995; 107: $382-388$

${ }^{26}$ Diaz O, Begin P, Torrealba B et al. Effects of noninvasive ventilation on lung hyperinflation in stable hypercapnic COPD. Eur Respir J 2002; 20: $1490-1498$

${ }^{27}$ Rasche K, Laier-Groeneveld G, Weyland W et al. Sauerstoffverbrauch der Atemmuskulatur unter kontrollierter bzw. assistierter Beatmung bei Patienten mit chronischer Ateminsuffizienz. Med Klin 1994; 89: S43-S46

${ }^{28}$ Vanpee D, Khawand CE, Rousseau L et al. Effects of nasal pressure support on ventilation and inspiratory work in normocapnic and hypercapnic patients with stable COPD. Chest 2002; 122: 75-83

${ }^{29}$ Garrod R, Mikelsons C, Paul EA et al. Randomized controlled trial of domiciliary noninvasive positive pressure ventilation and physical training in severe chronic obstructive pulmonary disease. Am J Respir Crit Care Med 2000; 162: 1335-1341

${ }^{30}$ Strumpf DA, Millman RP, Carlisle CC et al. Nocturnal positive-pressure ventilation via nasal mask in patients with serve chronic obstructive pulmonary disease. Am Rev Respir Dis 1991; 144: 1234-1239

${ }^{31}$ Lin CC. Comparsion between nocturnal nasal positive pressure ventilation combined with oxygen therapy and oxygen monotherapy in patients with severe COPD. Am J Respir Crit Care Med 1996; 154: $353-358$

32 Casanova C, Celli BR, Tost L et al. Long-term Controlled Trial of Nocturnal Nasal Positive Pressure Ventilation in Patients With Severe COPD. Chest 2000; 118: $1582-1590$

${ }^{33}$ Clini E, Sturani C, Rossi A et al. The Italian multicentre study on noninvasive ventilation in chronic obstructive pulmonary disease patients. Eur Respir J 2002; 20: 529-538 
${ }^{34}$ Elliott MW, Mulvey DA, Moxham J et al. Domiciliary nocturnal nasal intermittent positive pressure ventilation in COPD: mechanisms underlying changes in arterial blood gas tensions. Eur Respir J 1991; 4: $1044-1052$

${ }^{35}$ Schucher B, Baumann HJ, Hein $\mathrm{H}$ et al. The daily use of home mechanical ventilation and the change in $\mathrm{PaCO}_{2}$ in patients with COPD. Eur Respir J 2000; 16: 381s

${ }^{36}$ Windisch W, Vogel M, Sorichter S et al. Normocapnia during nIPPV in chronic hypercapnic COPD reduces subsequent spontaneous $\mathrm{PaCO}_{2}$. Respir Med 2002; 96: $572-579$

37 Elliott MW, Simonds AK, Carroll MP et al. Domiciliary nocturnal nasal intermittent positive pressure ventilation in hypercapnic respiratory failure due to chronic obstructive lung disease: effects on sleep and quality of life. Thorax 1992; 47: $342-348$

${ }^{38}$ Meecham Jones DJ, Paul EA, Jones PW et al. Nasal pressure support ventilation plus oxygen compared with oxygen therapy alone in hypercapnic COPD. Am J Respir Crit Care Med 1995; 152: 538 - 544

${ }^{39}$ Gay PC, Hubmayr RD, Stroetz RW. Efficacy of nocturnal nasal ventilation in stable, severe COPD during a 3-month controlled trial. Mayo Clin Proc 1996; 71: 533-542

${ }^{40}$ Goldberg A et al. Clinical indications for noninvasive positive pressure ventilation in chronic respiratory failure due to restrictive lung disease, COPD, and nocturnal hypoventilation - a consensus conference report. Chest 1999; 116: $521-534$

${ }^{41}$ Ferguson GT, Gilmartin M. $\mathrm{CO}_{2}$ rebreathing during BIPAP ${ }^{\circledR}$ ventilatory assistance. Am J Respir Crit Care Med 1995; 151: 1126- 1135

${ }^{42}$ Elliott MW. Noninvasive ventilation in chronic vebtilatory failure due to chronic obstructive pulmonary disease. Eur Respir J 2002; 20: $511-514$

${ }^{43}$ Criée CP. Ist Heimbeatmung wirksam? Pneumologie 2002; 56: $591-592$

${ }^{44}$ Windisch W, Freidel K, Matthys $\mathrm{H}$ et al. Gesundheitsbezogene Lebensqualität bei Patienten mit Heimbeatmung. Pneumologie 2002; 56: 610-620

45 Sivasothy P, Smith IE, Shnerson JM. Mask intermittent positive pressure ventilation in chronic hypercapnic respiratory failure due to chronic obstructive pulmonary disease. Eur Respir J 1998; 11: 34 - 40
${ }^{46}$ Schucher B, Hein H, Zuehlke IE et al. Noninvasive positive pressure ventilation improves quality of life in patients with COPD. Eur Respir J 2002; 20: 278s

47 Windisch W, Freidel K, Schucher B et al. Evaluation of health related quality of life using the MOS 36-Item Short Form Health Status Survey in patients receiving noninvasive positive pressure ventilation. Intensive Car Med 2003; 29: 615-621

${ }^{48}$ Perrin C, El Far Y, Vandenbos F et al. Domiciliary nasal intermittent positive pressure ventilation in severe COPD: effects on lung function and quality of life. Eur Respir J 1997; 10: 2835-2839

${ }^{49}$ Windisch W, Freidel K, Schucher B et al. The Severe Respiratory Insufficiency (SRI) Questionnaire: a specific measure of health-related quality of life in patients receiving home mechanical ventilation. J Clin Epidemiol 2003; 56: $752-759$

${ }^{50}$ Nocturnal oxygen Therapy trial group. Continuous or nocturnal oxygen therapy in hypoxemic chronic obstructive lung disease. Ann Intern Med 1980; 93: 191 - 198

${ }^{51}$ Report of the Medical Research Council Working Party. Long-term domiciliary oxygen therapy in chronic hypoxic cor pulmonale complicating chronic bronchitis and emphysema. Lancet 1981; 1: 681-685

${ }^{52}$ Schönhofer B, Barchfeld T, Wenzel M et al. Long term effects of non-invasive mechanical ventilation on pulmonary haemodynamics in patients with chronic respiratory failure. Thorax 2001; 56: 524-528

${ }^{53}$ Wijkstra PJ, Lacasse Y, Guyatt GH et al. A Meta-analysis of nocturnal noninvasive positive pressure ventilation in patients with stable COPD. Chest 2003; 124: 337-343

${ }^{54}$ Schucher B, Hein H, Baumann HJ et al. Nebenwirkungen der Heimbeatmung. Pneumologie 1999; 53: S77

55 Jones SE, Packham S, Hebden M et al. Domiciliary nocturnal intermittent positive pressure ventilation in patients with respiratory failure due to severe COPD: long term follow up and effect on survival. Thorax 1998; 53: $495-498$

${ }^{56}$ Schönhofer B, Köhler D. Akzeptanz und Effektivität der intermittierenden Selbstbeatmung bei COPD in der Initialphase der Therapieeinstellung. Atemw Lungenkrkh 1997; 23: 517-522 